\title{
The Initial Results of the Chung-Li Dynasonde for RF Environment Surveillance and Ionospheric Observations
}

\author{
Tung Yuan Hsiao ${ }^{1,2, *}$, Lung-Chih Tsai ${ }^{2,3}$, and Frank Tom Berkey ${ }^{4}$ \\ ${ }^{1}$ Department of Information Technology, Hsing Wu College, Lin-Kou, Taiwan, ROC \\ ${ }^{2}$ Institute of Space Science, National Central Uuiversity, Chung-Li, Taiwan, ROC \\ ${ }^{3}$ Center for Space and Remote Sensing Research, National Central University, Chung-Li, Taiwan, ROC \\ ${ }^{4}$ Institute of Physics, Utah State University, Logan, UT 84322-4145, USA
}

Received 7 September 2006, accepted 20 September 2007

\begin{abstract}
In 2004, a NOAA (National Oceanic and Atmospheric Administration) MF/HF radar or dynasonde was set up by the National Central University in Chung-Li $\left(24.5^{\circ} \mathrm{N}, 121.0^{\circ} \mathrm{E}\right)$, Taiwan. The Chung-Li dynasonde employs an interferometric array (including four receivers) for receiving ionospherically reflected echoes, and is a fully digital research ionosonde, capable of providing high precision spatial and temporal ionospheric measurements. Utilizing a pulse set defined by four pulses transmitted at two closely spaced frequencies, the measured parameters are the time-of-flight and sixteen complex-amplitudes. Two types of observations are conducted by the Chung-Li dynasonde - an active sounding mode and a passive sounding mode. The methods are utilized in observing the local ionosphere and RF environment above Chung-Li. The active sounding is capable of transmitting HF radio pulses for remote sensing of the ionosphere. Initial results from this study have revealed various special events for observing sporadic $\mathrm{E}$ and spread $\mathrm{F}$, along with the distribution of foF2 in a single day. These initial results will be used to confirm the surveillance of the ionosphere from other instruments, and conduct statistical comparisons between seasons. On the other hand, passive sounding receives and records signals from RF environments. It is used to monitor the $\mathrm{MF} / \mathrm{HF}$ band in real time, and is contrasted with the active results when the echo is weak.
\end{abstract}

Key words: Ionosphere, Dynasonde, Ionosonde, Passive radar, HF radar

Citation: Hsiao, T. Y., L. C. Tsai, and F. T. Berkey, 2008: The initial results of the Chung-Li dynasonde for RF environment surveillance and ionospheric observations. Terr. Atmos. Ocean. Sci., 19, 515-524, doi: 10.3319/TAO.2008.19.5.515(AA)

\section{INTRODUCTION}

Passive radar is a technique for observing targets of interest via radio signals that are already present in the environment. By intercepting both the signals radiated by a transmitter and/or scattered from targets of interest, it is possible to perform radar measurements on signal range, Doppler frequency shift, and bearing. The accuracy depends on the nature of the illuminating waveform and the characteristics of the passive radar system. In most cases, the $\mathrm{MF} / \mathrm{HF}$ signals are generated by FM or AM radio station transmissions. Such illuminators are present throughout most of the world. They have a strong propagating energy, and often transmit signals that are well suited for radar applications. The technique can also be applied to signals

* Corresponding author

E-mail:TungYuanHsiao@gmail.com from a cooperative illuminator, such as an active radar system. In this case, it is more similar to traditional radars. Many investigations in the requirements and potential of passive radar have been made, yet only a few are publicly available (Ringer et al. 1999). Experimental systems have been described in using UHF television for aircraft tracking (Howland 1994), or in using FM radio signals for ionospheric investigations (Sahr and Lind 1997). For ionospheric radio science, passive radars are also useful for observing E-region irregularities (Lind et al. 1999). In general, there are two types of plasma instabilities that are capable of exciting small scale irregularities in E region. One is two-stream instability and the other one is gradient-drift instability. Information on Doppler velocity is necessary to identity types of $\mathrm{E}$ layer irregularities. Type one irregularities are created at altitudes between 95 and $120 \mathrm{~km}$ 
by two-stream instability, which occurs between sufficiently strong electric fields. Basically, the phenomenon will cause sporadic E, which comes from the traces of Es echoes in ionograms. They commonly occur in association with the aurora surrounding the Earth's poles, with the equatorial electro-jet at the equator and occasionally at the mid-latitudes. A summary of the phenomenon, theory, and experimentation for the aurora regions is available ( $\mathrm{Sahr}$ and Fejer 1996). The passive HF radar method has been utilized in ionospheric experiments for diagnosing both natural and "artificial" ionospheric irregularities, which are found to provide indispensable data. With this method, supplemented by other diagnostics, it was recently discovered that auroras can be triggered when strong radio waves interact with lower layers of the ionosphere (Blagoveshchenskaya et al. 1999). As this is the first evidence of an anthropogenic ionosphere-magnetosphere coupling, it is particularly important to study the phenomena further.

Environmental monitoring by radio methods of this type are expected to be increasingly important in the future. A modern ionosonde, such as a dynasonde, has the potential to perform well as passive radar. The basic technology of the dynasonde interference phased array is appropriate for passive radar. The dynasonde employs a $0.2-20 \mathrm{MHz}$ frequency range under a passive mode. A baseline of up to $2000 \mathrm{~km}$ is conceivably workable for receiving one- or multi-hop echoes reflected from the ionosphere. Large baselines will require interception of the transmitter signal via a separate receiver system close to the point of transmission. As pointed out previously, ultra-stable HF radio signals in the $10-30 \mathrm{MHz}$ range have already been used for passive radar investigation of ionospheric irregularities. The final location of the dynasonde will have a large impact on what types of ionospheric phenomenon are observable, when it is used as passive radar. Ionospheric scintillations, due to Eregion irregularities, will be a source of interference for radio applications (Vats et al. 1995).

\section{THE SYSTEM OVERVIEW}

Figure 1 presents a simplified block diagram of the existing Chung-Li dynasonde. The Chung-Li dynasonde can be separated into four general sections: (a) system control and timing (handled by a PC); (b) the radio frequency transmitter and receivers; (c) data acquisition and reduction (handled by a PC); and (d) the associated antennas. The most important component is the analogue section comprised of the transmitter and the receivers; of these, it is the receiving section that defines the capabilities of the Chung-Li dynasonde. The Chung-Li dynasonde employs four receivers so that simultaneous measurements of phase can be carried out from different receiving antennas (so that a position 'fix' can be carried out). A general overview of the dynasonde RF hardware is shown in Fig. 2. There are two parts for RF hardware. One is the transmitter and the other is the receiver. The transmitter and receivers are frequency agile and can operate from 0.1 to $30 \mathrm{MHz}$. The transmitter is capable of delivering $60 \mu$ s pulses at a pulse repetition rate of 100 pulses per second and with a peak power of $18 \mathrm{~kW}$. A data bus is controlled by a PC to step the AGC gain (one step is $4 \mathrm{db}$, to-

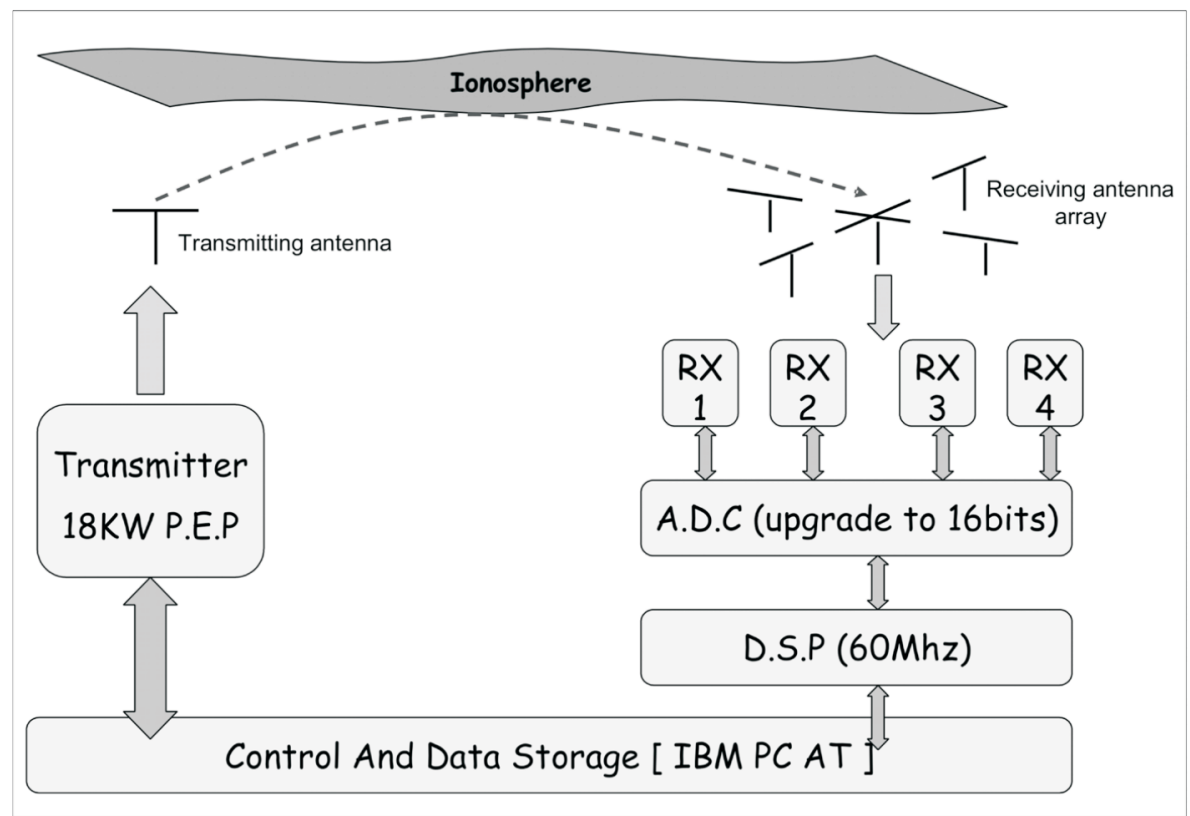

Fig. 1. A simplified block diagram of the Chung-Li dynasonde. The timing control, frequency control, ADC, and DSP tasks are handled by a personal computer. The pulsed RF signals transmitted by a solid-state transmitter and received by 4 receivers, are processed by a DSP card and then saved to an appropriate mass storage device. (Hsiao 2002). 


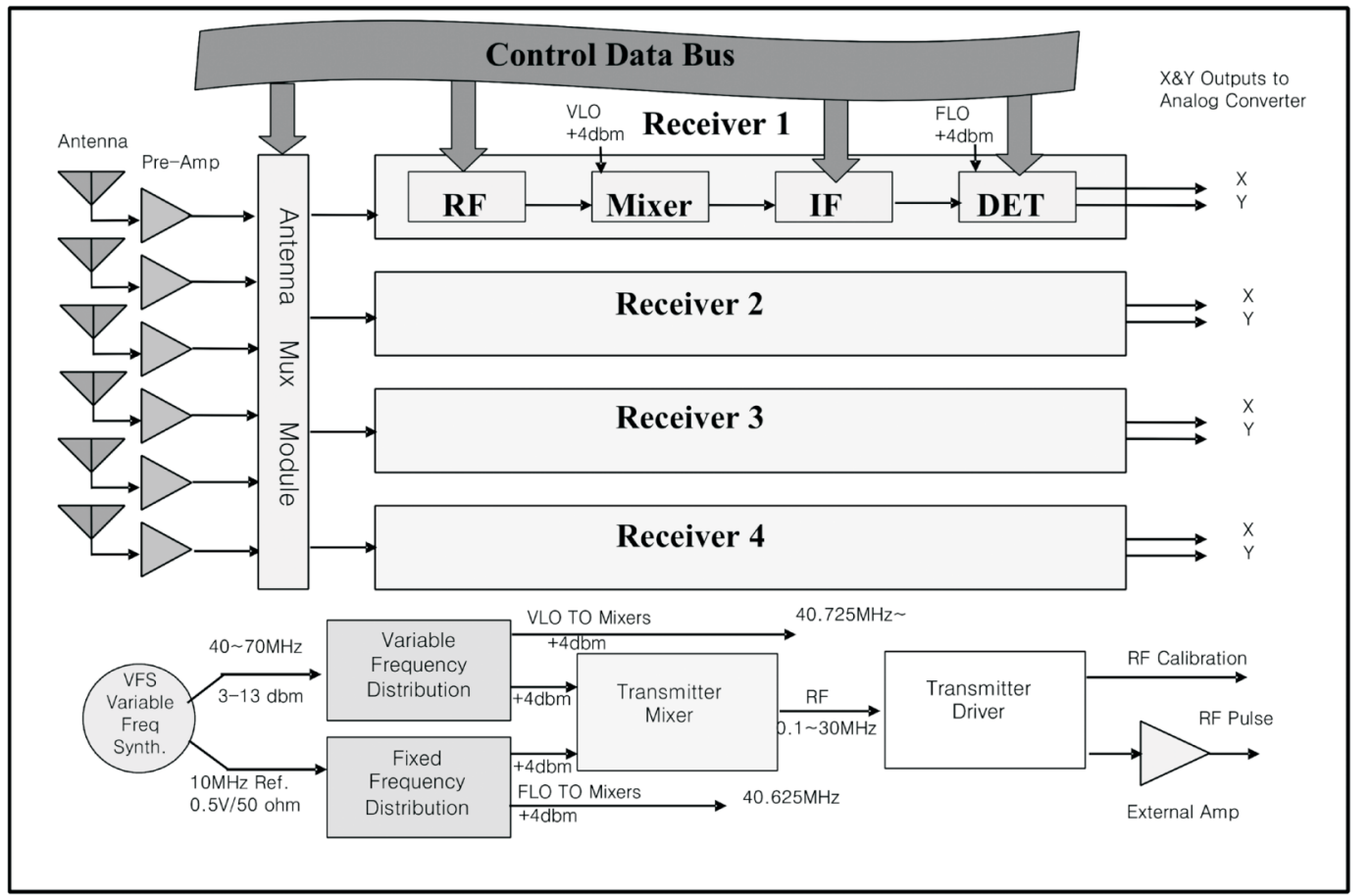

Fig. 2. There are two parts for the RF hardware. One is the transmitter and the other the four receivers. The transmitter and receivers are frequency agile and use a base-band frequency of $40.625 \mathrm{MHz}$. The PC controlled frequency synthesizer generates pulses of $40.725 \sim 70.625 \mathrm{MHz}$, which are down converted to the range 0.1 to $30 \mathrm{MHz}$. (Hsiao 2002).

tally $120 \mathrm{db}$ ) and the bandwidth $(1,10$, or $30 \mathrm{KHz})$. The function of superheterodyne is employed at $40.625 \mathrm{MHz}$ base band, and the PC controls the variable frequency synthesizer, which varies from 40.725 to $70.625 \mathrm{MHz}$, to generate a signal that varies from $0.1 \sim 30 \mathrm{MHz}$.

Recent results from the Chung-Li dynasonde include the acquisition of both active and passive soundings. Two sounding modes have been employed as routine operations starting in March 2006. The data can be reviewed and/or fetched from the following URL (http://140.115.111.226/ database/).

\subsection{Data Processing and Echo Detection}

In the Chung-Li dynasonde system, it was necessary to upgrade the DSP card to approach a better sampling resolution and also because the original DSP card was no longer available. Our dynasonde utilizes a DSP card termed "PC32", and the PC32 board improves the sampling resolution from 12 to 16 bits and utilizes floating point calculations rather than integer (Black and Hsiao 2004). Another advantage is its immunity to noise. Digital signal processing is used to determine the ionospheric echoes of pulses and to exclude spurious radio interference (e.g., from broadcast stations). Two techniques are used for this. First, the digitized outputs from the receivers are scanned to see if the received values exceed a designed threshold and to fit the expected pattern of echo as a Guassian shape as shown in Fig. 3. Secondly, rather than transmitting a single pulse, the Chung-Li dynasonde transmits a set of four pulses and only identifies echoes if these pulses are received at the same time of flight. Because each of the receivers measures I and Q components as outputs, there are 32 possible digital data values ( 4 pulses by 4 receivers by 2 components) to be recognized within each transmitted pulse set. Both of these echo recognition functions taken together are termed 'peak fitting' and are carried out by a program executed in the PC32 card. Note that one PC32 card only has four input signal processing channels, and two PC32 cards are used to service four receivers. Receiver complex-amplitude output is sampled frequently at $10 \mu$ intervals in each channel, throughout a wide range of echo delay, whether or not echoes are present. The method of echo recognition (Wright and Pitteway 1979) occurs in real time by a process (peak) of range coincidence: echo amplitude peaks are detected within the complex-amplitude samples by a 5- or 7-point algorithm. Putative echoes from each of the $n$ pulses of the pulse set are compared for coincidence within a small tolerance, and those which yield $n$-hits are accepted as genuine echoes. In some observational modes (more detail in section 3), $m$ hits (with $n>m$ ) are accepted. For $m=n$, and $n=4$ the probability of accepting a false echo is less than $10^{-4}$. However, the process discards some genuine echoes; in particular, this happens when two echoes are somewhat overlapped, and when their relative phases are not nearly opposite, whereupon their shape of amplitude may resemble a broad plateau, or a peak plus a plateau. 

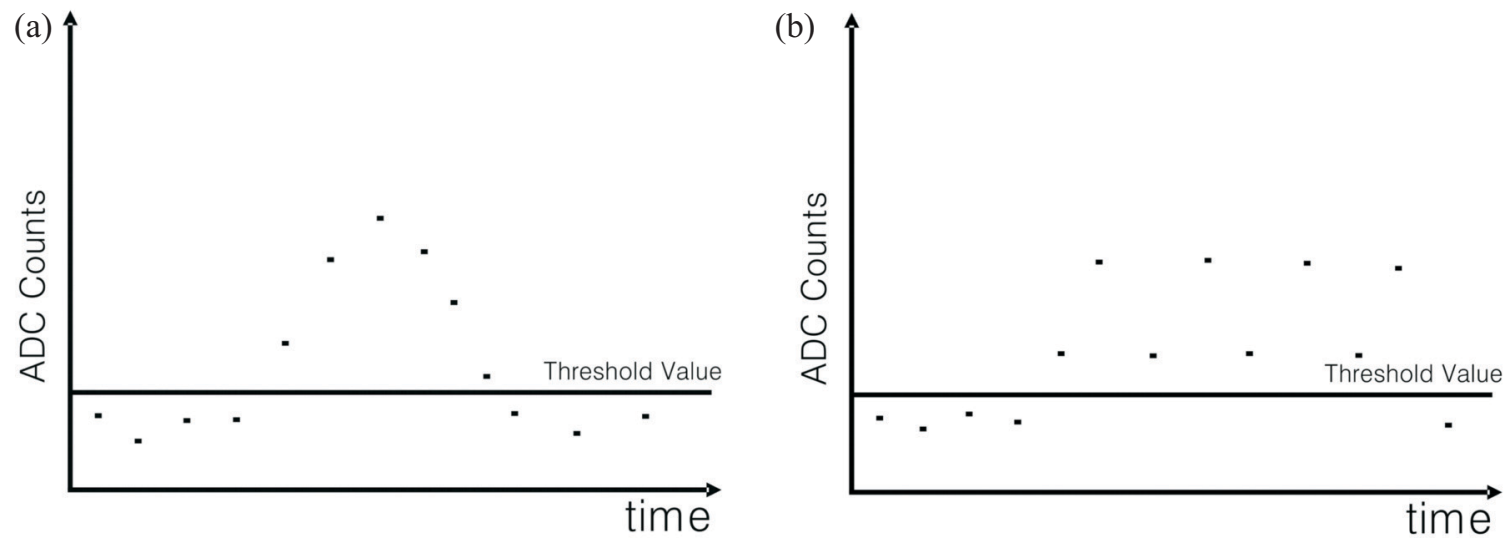

Fig. 3. Echo detection methodology. Figure 3a is a typical example of peak detection by the DSP card. The pulse width sampled at $100 \mathrm{KHz}$ is $60 \mathrm{mi}-$ croseconds. The DSP card samples a window of 80 micro-second width and searches amplitude peaks of echoes. The normal echoes have typical Gaussian shapes as shown in Fig. 3a. Figure 3b shows an example which cannot be identified as a peak after DSP even if the ADC counts exceed the designed threshold (Referred from Berkey and Rose 1998).

\subsection{Pulse Set and Receiving Antenna Configuration}

From the design of the transmitted pulse set for the Chung-Li dynasonde, we can calculate all the echo parameters from the phases measured in one pulse set $\left(\Phi_{1} \sim \Phi_{\mathrm{n}}\right)$ (Pitteway and Wright 1992; Tsai et al. 1993). The arrangement of receiving antennas is shown in the Fig. 4. The north-south orientated antenna is not aligned with the geographic north but the magnetic north. The echo parameters from phase measurements include $\Phi_{\mathrm{x}}$ and $\Phi_{\mathrm{y}}$, which are phase differences caused by antenna separations and are measured as positive values in the $\mathrm{x}$ and $\mathrm{y}$ directions, respectively, from the receiving antenna array centre; $\Phi_{\mathrm{p}}$, which shows the effect of different antenna orientation and thus enables the polarization of the echo to be identified as "ordinary" or "extraordinary" (normally \pm 90 degrees); $\Phi_{t}$, which shows the effect of temporal changes during a pulse set and is measured in degrees per inter-pulse interval; $\Phi_{\mathrm{f}}$, which is the effect of sounding frequency changes and is used to stationary phase ranging to approach a better range resolution of 100 meters; and $\Phi_{0}$, which is the average of the two phases measured for the echo on the first pulse. The user can change the configuration of the sounding pulse set and arrange the receiving antennas for different user requirements. The echo characteristics are determined by the interference of signals from spaced antennas, interval pulses within set, and different sounding frequencies. Figure 4 shows the design of receiving antenna array and pulse set pattern used by the Chung-Li dynasonde system. All of the simulated characteristics of the phase parameters have a $2 \pi$ phase ambiguity and the values of relative confidence limit factor (RCLF) are computed to be $0.5306,0.4082,0.3536,0.2240,0.5088$, and 0.5054 for $\Phi_{0}, \Phi_{\mathrm{x}}, \Phi_{\mathrm{y}}, \Phi_{\mathrm{t}}, \Phi_{\mathrm{p}}$, and $\Phi_{\mathrm{f}}$, respectively.

\section{SOUNDING CONFIGURATIONS}

There are several sounding configurations for the
The Chung-Li Cross-WERPOL Receiving Array

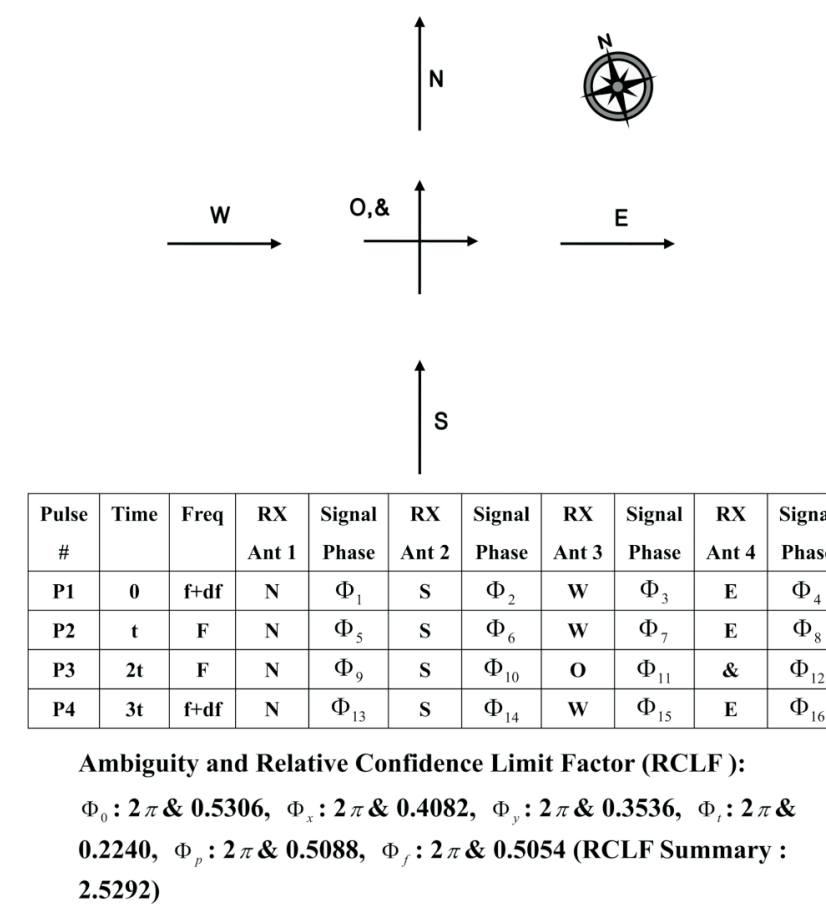

Fig. 4. The pulse set and the receiving antenna array for the Chung-Li Cross-WERPOL. There are four receivers, six antennas and four pulses to accompany a pulse set. $\mathrm{P} 1$ is pulse one of a pulse set; and $\mathrm{t}$ is the time of the next transmitted pulse. The positions of W, S, E, N, O and \& are the locations of antennas. There are totally sixteen phases in one pulse set to find the ionospheric parameters.

Chung-Li dynasonde operations and the user can design sounding configurations for their particular observational program. Standard configurations that employ frequency shifting include B, I, K, and P modes. In I (or Ionogram) mode the radar usually sounds on a large number of frequencies in a single sweep from low to high frequencies. 
The increment between every two frequencies can either be constant (a linear sweep is shown in Fig. 5a) or a logarithmic step increased when the sounding frequency are increased (a logarithmic sweep is shown in Fig. 5b). B mode also sweeps the frequency range but repeats several times in each set of frequencies or frequency ramps. It consists of an "initial" ramp of frequencies (as I mode) with smaller ramps superimposed on it. B mode is capable to give a wide variety of possible sequences of frequencies. For example, each ramp may have a linear (as shown in Fig. 5c) or logarithmic (as shown in Fig. 5d) increase in gradient; the same ramp may be repeated more than once before moving on to a higher frequency ramp (as shown in Fig. 5c); the frequency spacing between consecutive ramps may be constant or be increased logarithmically (as shown in Fig. 5e). The K (or Kinesonde) mode sounds on a limited number of frequencies supplied from a user-generated table. Each frequency is repeated to sound a number of times (as shown in Fig. 5f). The $\mathrm{P}$ Mode sounding also sounds on a limited number of frequencies in the same way as a $\mathrm{K}$ mode, but the system program returns the "raw" ADC data (no echo identification and peak searching algorithms employed in the DSP board). However, digitization needs to be constrained to a limited height range to keep the amount of data generated within practical limits.

\subsection{The Active Sounding Results}

Active sounding transmits powered pulses to remotely sense the ionosphere. We started routine surveillance in April 2006 utilizing B-mode sounding. The example ionograms for daytime and nighttime observations are shown in
Figs. $6 a$ and $b$, respectively. There are several gaps in the frequency domain in these ionograms. We double check the passive sounding results to see if the frequency ranges are being used by any radio station, and the transmitting power is strong enough to interfere with echo detection of the Chung-Li dynasonde. Another use of these active and passive sounding results is choosing frequency bands to approach better communication quality. Figures $6 \mathrm{c}$ and $\mathrm{d}$ present special events of sporadic E and spread F, which represent concentrated ionospheric research. The critical frequency of sporadic E (foEs) is highly variable in time and space. Recent research results show sporadic E events can be caused by ionospheric wind shear, thunderstorms, weather, meteor or geomagnetic activity (Mathews 1998). The sporadic $\mathrm{E}$ close to the equator is essentially a daytime phenomenon, where there is little variance with seasons. But in the aurora area, sporadic E usually appears during nighttime, where it also exhibits a weak variance with seasons. The foEs of the mid-latitudes has been depicted as being less than that of aurora areas according to earlier research (Davies 1990). However, in the case of sporadic E over Chung-Li, which is near an area where electro-jets descend, something quite distinct appears to be occurring; the results of Bowman and Mortimer (2002 and 2003) support the existence of coupling between isolated spread- $F$ events and sporadic $E$ enhancements for the EIA crest station especially for post-sunset periods of sunspot maximum years from old Chung-Li data. From the initial results of the Chung-Li dynasonde, sporadic E appeared in both the daytime and nighttime. Figure 7 presents foEs and fminEs profiles obtained on 28 May 2006. Sporadic E is shown as occurring throughout most of the day. The daytime foEs is (a)

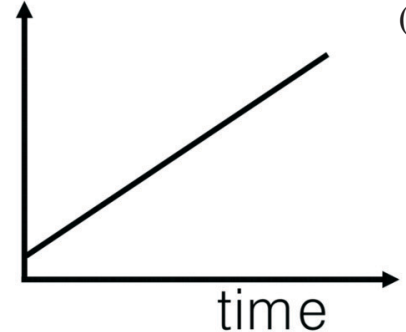

(d)

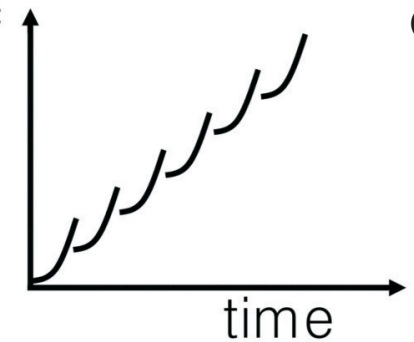

(b)

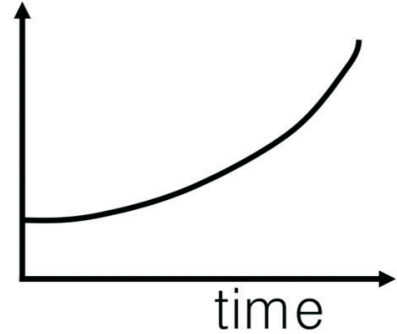

(e)

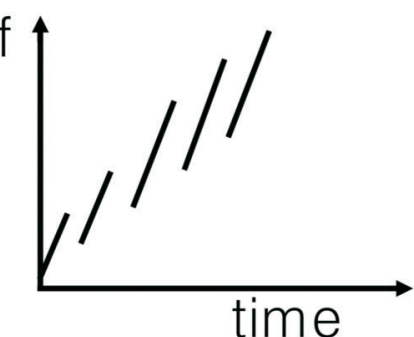

(c)

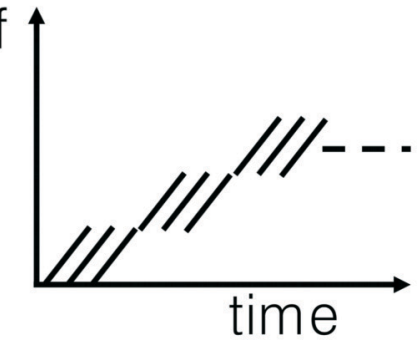

(f)

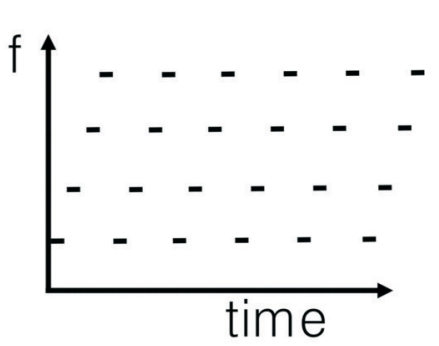

Fig. 5. Sounding modes for the Chung-Li dynasonde. (a) linear sweep-figure of I mode sounding; (b) logarithmic sweep-figure of I mode sounding; (c) linear sweep-figure of B mode sounding; (d) logarithmic sweep-figure of B mode sounding; (e) the frequency spacing between consecutive ramps increasing logarithmically of B mode sounding and; (f) the K mode sounding (Referred from Berkey and Rose 1998). 

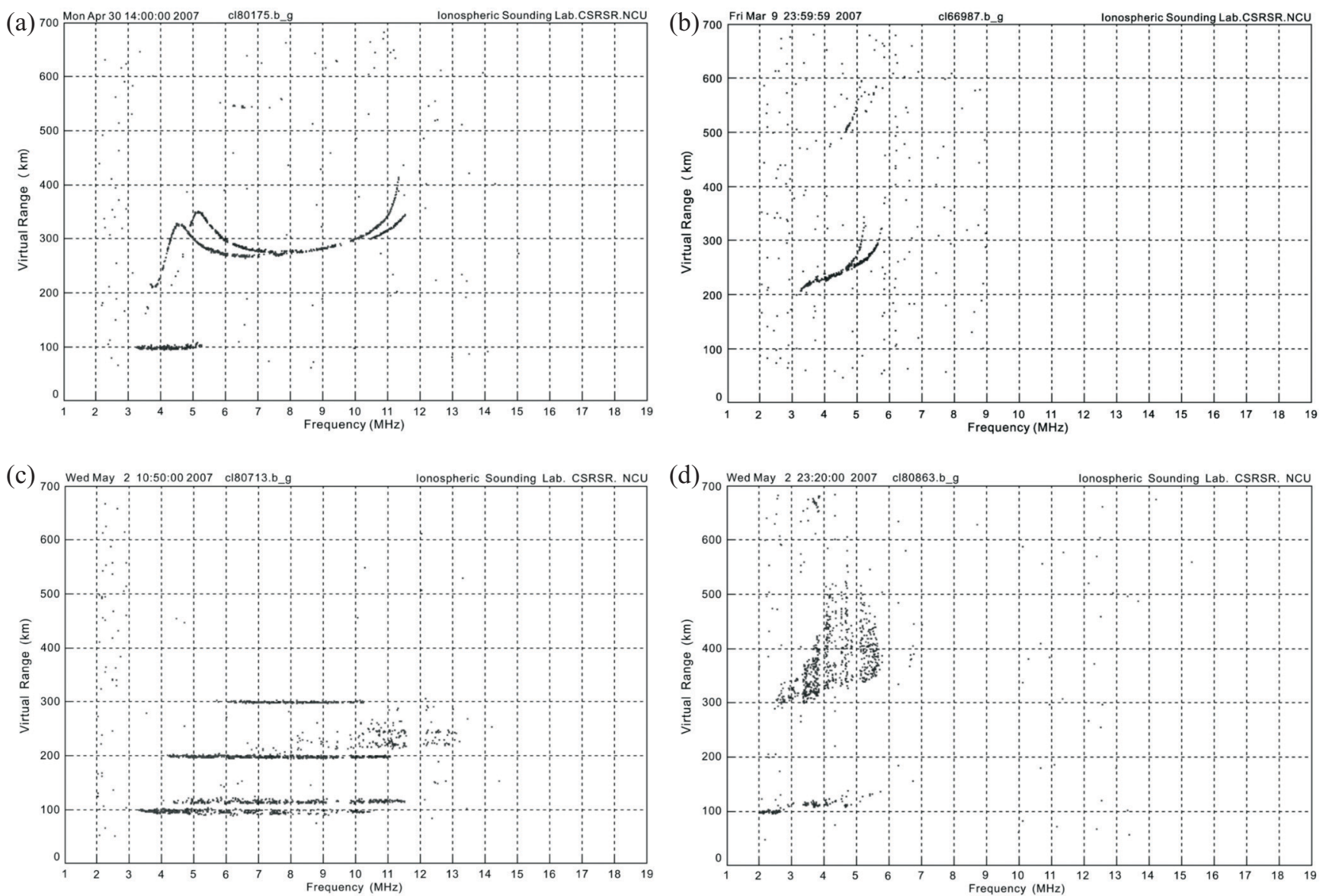

Fig. 6. (a) A B-mode sounding ionogram during daytime; (b) A B-mode sounding ionogram during nighttime; (c) A special event for the sporadic-E layer; (d) A special event for Spread F.

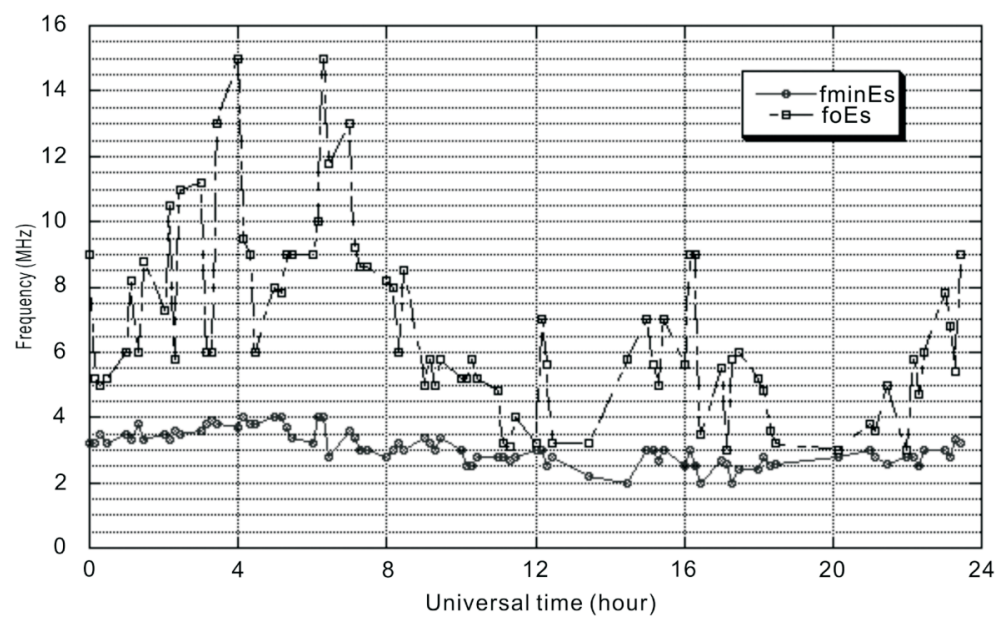

Fig. 7. The diurnal profiles of foEs and fminEs on 28 May 2006. The fminEs did not change much for this day. foEs was much more active and has the highest values at noon and midnight.

higher than the nighttime foEs, and the foEs is stronger at noon and midnight in contrast to the morning and evening, respectively. Moreover, another sporadic E activity observed by both a satellite beacon receiver and the dynasonde at Chung-Li are presented in the Fig. 8. Figure 8a showed a typical event on 3 May 2007, whereby the beacon signal at $150 \mathrm{MHz}$ was disturbed and the $400 \mathrm{MHz}$ beacon signal was not. Meanwhile, the Chung-Li dynasonde observed strong sporadic $\mathrm{E}$ at $1820 \mathrm{LT}$ on the same date as shown in Fig. $8 \mathrm{~b}$. We conclude that the signal of $150 \mathrm{MHz}$ was more sensitive to sporadic $\mathrm{E}$ than the signal of $400 \mathrm{MHz}$. More statistical data from the Chung-Li dynasonde data is required to identify sporadic E and to perform seasonal comparisons in future works.

In another event, we observed both sporadic $\mathrm{E}$ and spread $\mathrm{F}$ as shown in Fig. 9 recorded by the Chung-Li dynasonde and tri-band beacon receiver on 27 April 2006. Scintillation happened at both 150 and $400 \mathrm{MHz}$ beacon signals as shown in Fig. 9a. Figure 9b presents a Chung-Li ionogram recorded at 1250 LT of 27 April 2006, and shows a strong spread $\mathrm{F}$ but weak sporadic $\mathrm{E}$ event on the same date. The spread F occurrence mainly exists in two areas: (1) equatorial regions, invariably at night, and (2) high latitude regions mostly at night, but sometimes 
(a)

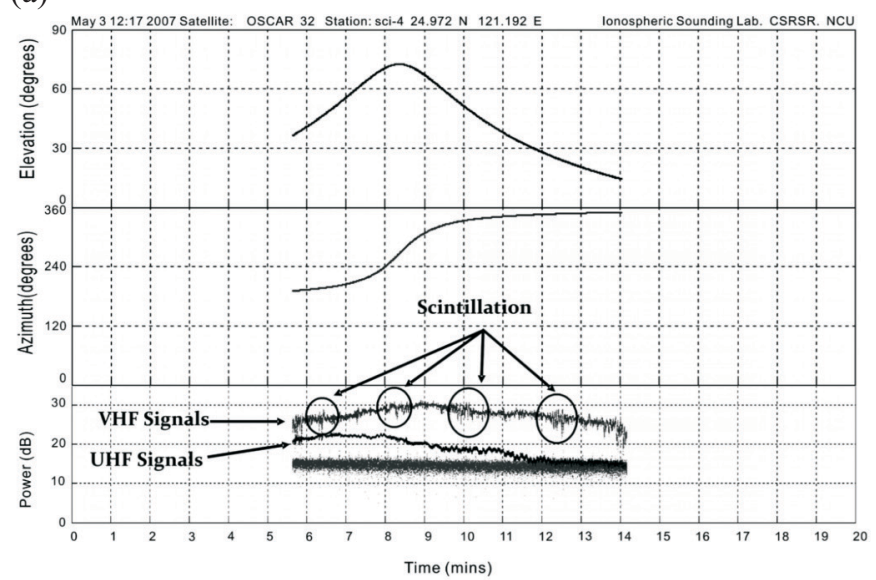

(b)

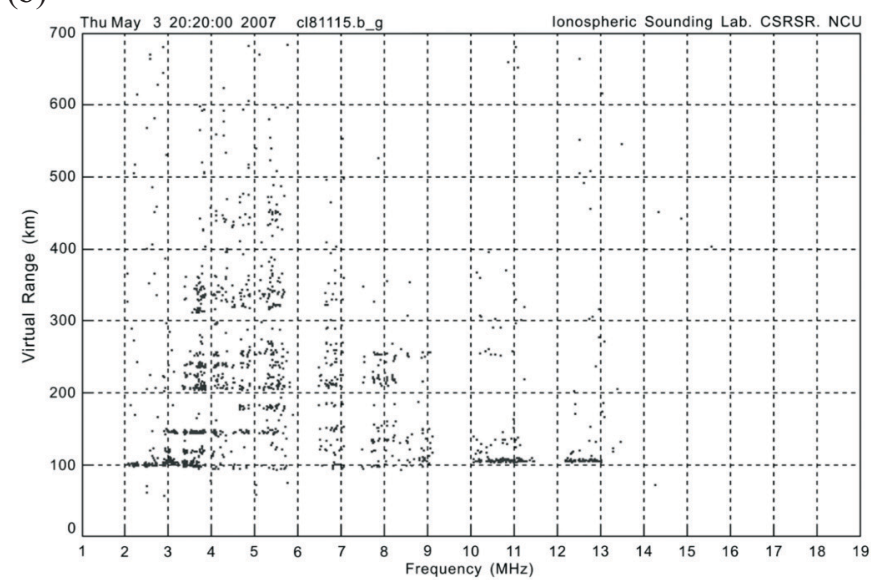

Fig. 8. (a) A special event for the tri-beacon satellite data of the Chung-Li station at 2017 LT of 3 May 2007. There are three graphs, which include azimuth angle, elevation angle, and the power of 150,400 , and $1066.3 \mathrm{MHz}$ signals. We determine $150 \mathrm{MHz}$ scintillation signals via the circuits. Meanwhile, the $400 \mathrm{MHz}$ signals did not appear as scintillations. (b) The ionogram recorded above Chung-Li at 2020 LT of 5 May 2007.

(a)

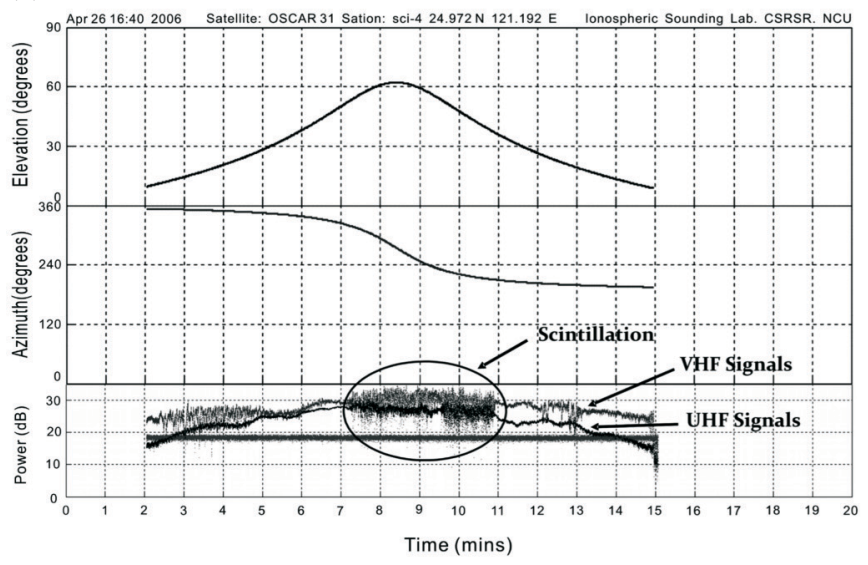

(b)

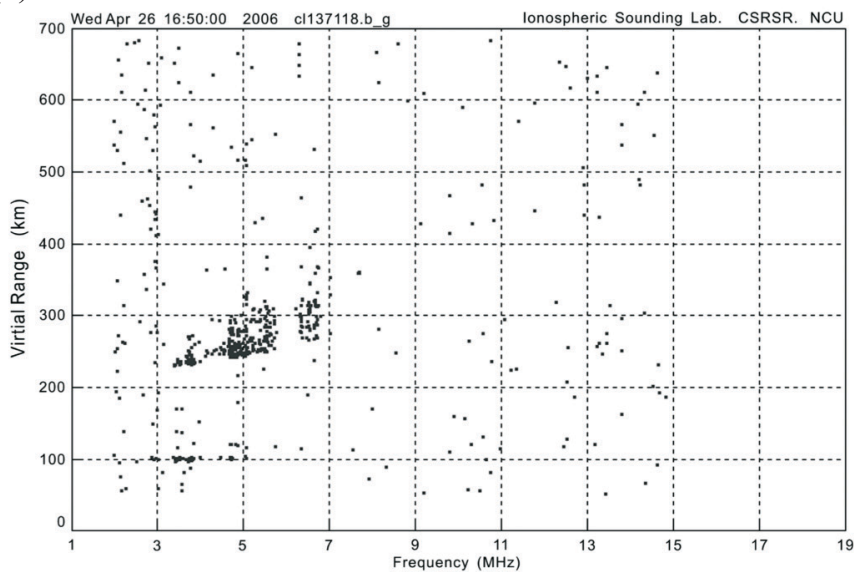

Fig. 9. (a) A special event for the tri-beacon satellite data of the Chung-Li station at 1246 LT of 27 April 2006. We also determine the scintillation signals in the circuit, but scintillation occurs at both 150 and $400 \mathrm{MHz}$ beacon signals. (b) The ionogram of Chung-Li at 1250 LT of 27 April 2006 . There is a strong Spread F event at this time.

during the day as well. Spread $\mathrm{F}$ is caused by the scattering of signals from irregularities embedded in the ionosphere, both within and away from the zenith. Spread F above Chung-Li belongs to equatorial spread $\mathrm{F}$, which usually occurs in latitudes bounded by $\pm 20^{\circ}$.

Diurnal foF2 profiles from the Chung-Li dynasonde are shown in Fig. 10. The F2-layer critical frequency foF2 is an important figure that gives an indication of the state of the ionosphere. It is obtained by sending a signal pulse directly upwards. As the frequency is increased a point is reached where the signal will pass right through the layer, and on to the next one, or into outer space. The frequency at which this occurs

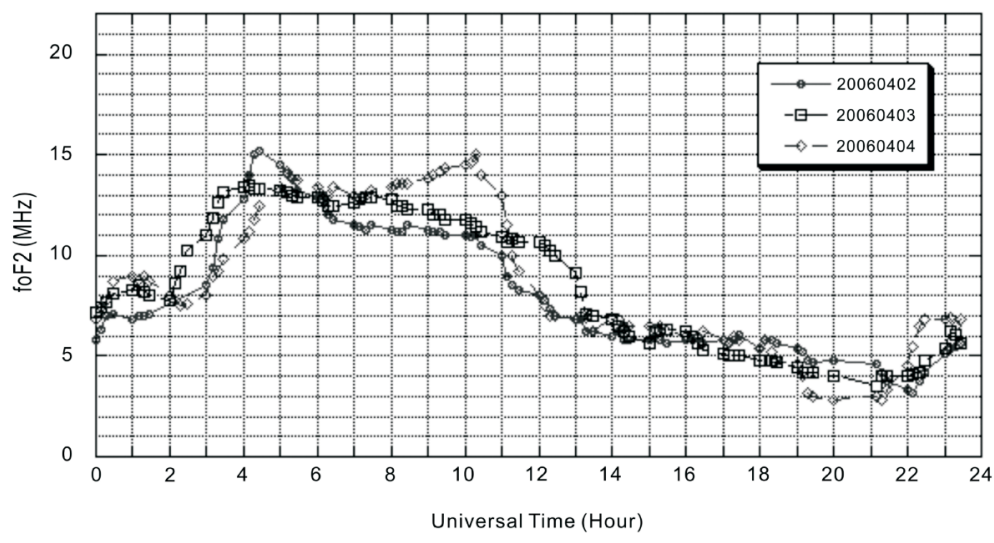

Fig. 10. The diurnal profiles of foF2 from 2 to 4 April 2006. The three days are close, so the diurnal variation of the ionospheric critical frequency of the F2 layer did not change much. The peak in foF2 was close to UT 4. It increased from UT 2 to 4 and then started to fall off during evening from about UT 10 to 14. 
is called the critical frequency. This will give an indication of the state of the ionosphere for that area of the world. The F2 layer is so far the most important layer, and it is also the most unstable from an $\mathrm{HF}$ radio communication point of view. It follows Snell's law either diurnally or seasonally. The distribution of foF2 within one day is shown in Fig. 10 from 2 to 4 April 2006. Not much change was seen during these three days. foF 2 increased quickly around 0400 UT (1200 LT) to about $14 \mathrm{MHz}$, and started to decrease fast at 1000 UT (1800 LT). This means the ion charging of the ionosphere took place from $0200 \mathrm{UT}$ (1000 LT) to $0400 \mathrm{UT}$ (1200 LT) when foF 2 was at peak of $14 \mathrm{MHz}$ and then began to decline to reach an end point for ion charging at $1000 \mathrm{UT}$ (1800 LT) to 1300 UT (2100 LT) when it fell to $5 \mathrm{MHz}$. Further work needs to be done on foF 2 in low latitudes for different seasons and diurnal time periods.

\subsection{The Passive Sounding Results}

Radio waves can be transmitted from all over the world and be monitored by adequate radio receivers. The ChungLi dynasonde usually operates passive sounding and then active sounding processing every fifteen minutes. An ex- ample result of passive sounding is shown as the frequency spectrum of Fig. 11. The color represents the signal amplitude from 55 to $70 \mathrm{~dB}$. The recording time is 1803 UT (i.e., 0203 LT) of 10 May 2006. The operating frequency scans from 1.5 to $20 \mathrm{MHz}$ individually for 2000 frequencies. We recorded a set of 50-bin data in 16-bit resolution at each frequency. We abandoned frequencies of less than $1.5 \mathrm{MHz}$, because the frequency band is too close to AM band used for broadcasting from $800 \mathrm{~K} \sim 1.4 \mathrm{MHz}$. There are eight strong communication bands between 1.5 to $20 \mathrm{MHz}$ in the diurnal RF environment surveillance results as shown in Fig. 12. The RF surveillance spectrums are different during the daytime and night time. A daily cycle roughly can be seen. We classify the eight comparatively strong background signals in eight bands in Fig. 12. They are "A", "B", "C", "D", "E", "F", "G", and " $H$ " bands for the frequencies from 17 to 18,15 to 16,13 to 14,11 to 12,9 to 10,7 to 8,5 to 6 , and 3 to $5 \mathrm{MHz}$, respectively. Meanwhile, the eight bands could be classified into two representatives for day and night time, separately. During the daytime the background interference can reach up to $18 \mathrm{MHz}$ of band $\mathrm{A}$ from 4 to 10 for universal time and is strongest at band $\mathrm{B}(15$ to $16 \mathrm{MHz}$ ). Both bands A and B then decay and slowly disappear in the

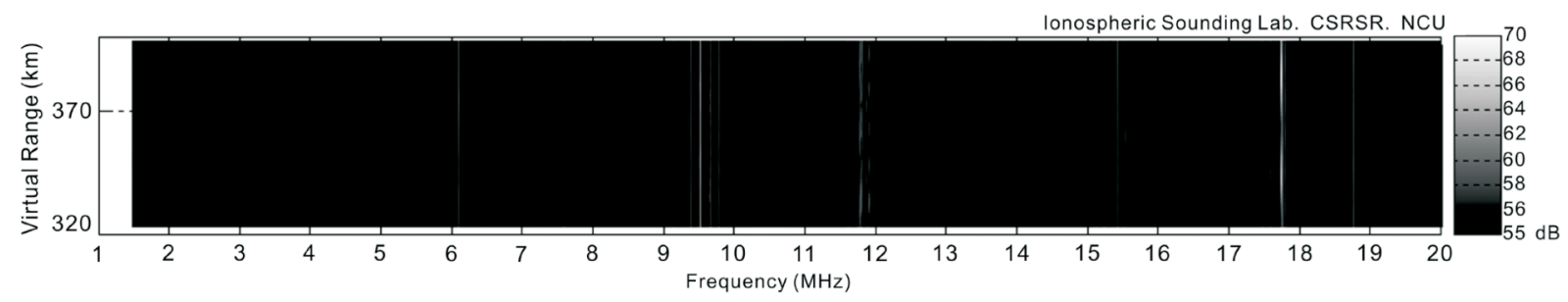

Fig. 11. A passive sounding example spectrum from 1.5 to $20 \mathrm{MHz}$ at 1803 UT of 10 May 2006.

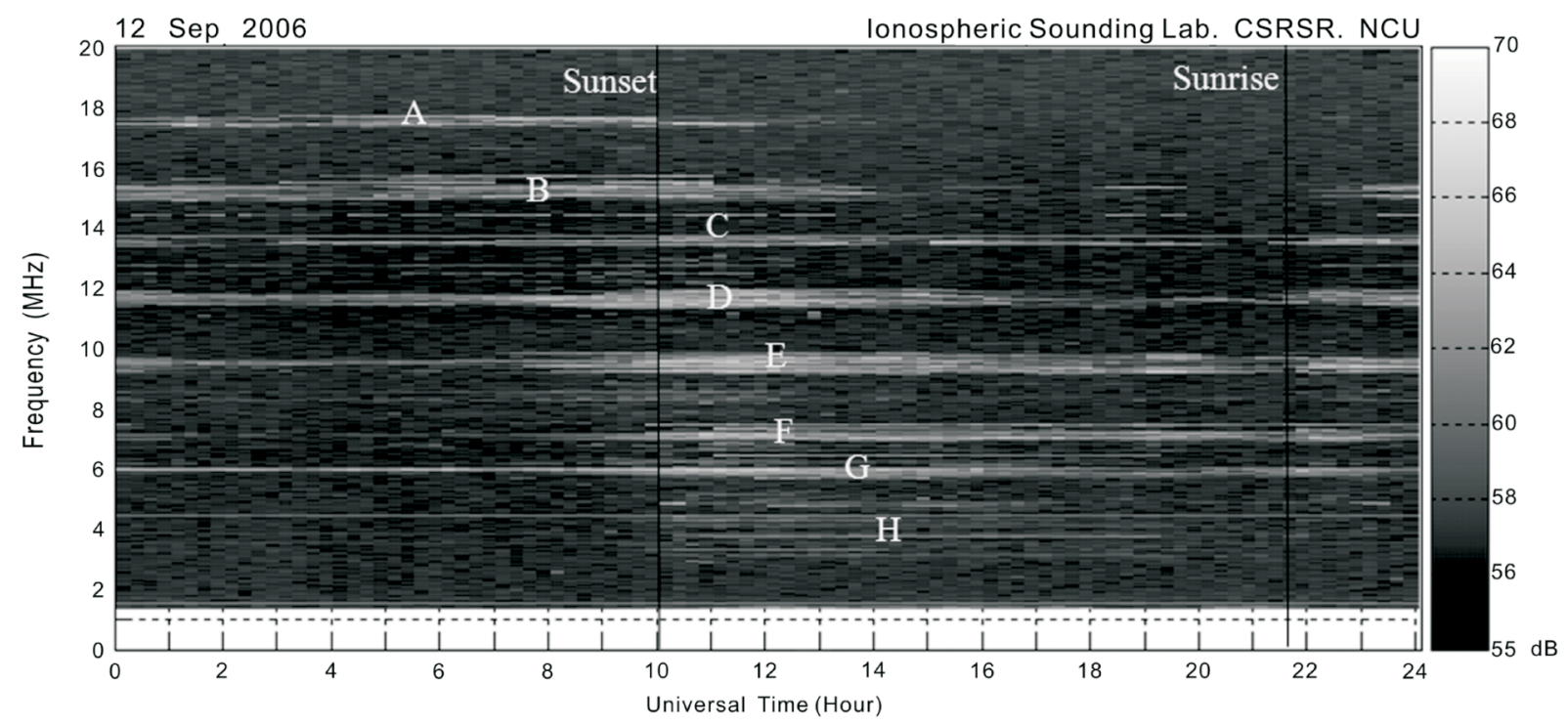

Fig. 12. The diurnal passive sounding spectrum of 12 September 2006 from 1.5 to $20 \mathrm{MHz}$. This result comes from passive soundings every 15 minutes (see Fig. 11). There were eight stronger interference bands, labeled from A to H, above Chung-Li. 
evening. On the contrary, the radio bands including the $\mathrm{C}$, $\mathrm{D}, \mathrm{E}, \mathrm{F}, \mathrm{G}$, and $\mathrm{H}$ bands between $2 \sim 14 \mathrm{MHz}$ are more active in the nighttime, where the strongest are bands $\mathrm{D}$ and $\mathrm{E}$ (11 $\sim 12$ and $9 \sim 10 \mathrm{MHz}$, respectively). The D- and E-band interferences increase during the evening and are strongest at $\sim 12$ UT (i.e., 20 LT) but decay during the daytime. Furthermore, the occurrence times for band interferences in the morning are about 21, 22, 23, and 24 UT (i.e., 5, 6, 7, and $8 \mathrm{LT}$ ) for bands $\mathrm{D}, \mathrm{C}, \mathrm{B}$, and $\mathrm{A}$, respectively, and are one-hour delayed for the higher band; the decay times for bands D, C, B, and A are about 13, 14, 15, and 16 UT (i.e., $21,22,23$, and 24 LT), which are also one-hour delayed for the higher band. With the result, we note that the interference variations are caused by ionospheric diurnal variation. Meanwhile, the $\mathrm{H}$ band interference is not stable and spreads from 3 to $5 \mathrm{MHz}$. It is sometimes more stable when sporadic E occurs. It can be concluded that there exists a close relationship between the RF environment and ionospheric conditions. We also present the differences in diurnal RF environment spectrums for different seasons in terms of when sunset and sunrise occur (see Fig. 13). ence. In our experience, strong interference covers echoes that reflect from the ionosphere in ionospheric observations. Hence we see the ionogram present with a blank band caused by such strong interference. Weak interference, however, does not cover the echo of active sounding but changes the actual phase of the echo. We may use passive sounding data to check the status of background noise when the recorded phases of active soundings are not continuous. Furthermore, from active sounding ionograms, sporadic E occurred typically in the morning and at noontime but it also sometimes occurred during the night. Other studies seem to exhibit a similar trend (Bowman and Mortimer 2002, 2003); however, more recorded data is needed to clarify statistical characteristics. Spread F usually occurs at night as in other regions. For future studies, we hope to confirm the FORMOSAT-3/COSMIC data and study seasonal and/or statistical characteristics for other applications.

Acknowledgements This work is supported by NSC942111-M-008-031-AP3 from the National Science Council of the Republic of China. We are also very grateful to the comments made by the reviewers during the revisions of this paper. (a)

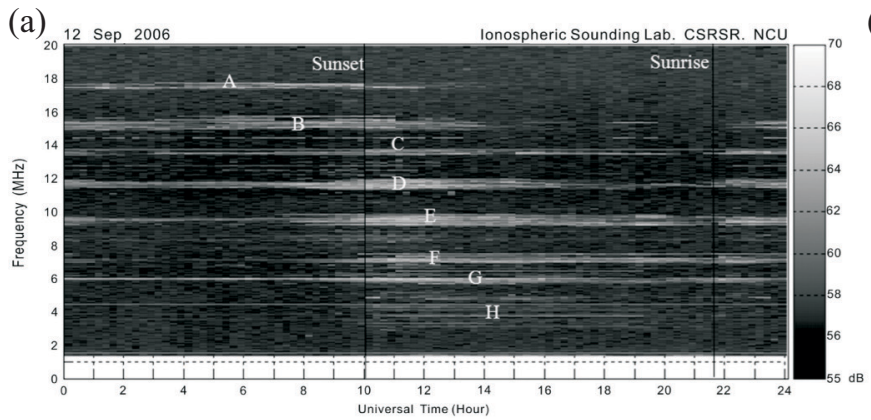

(c)

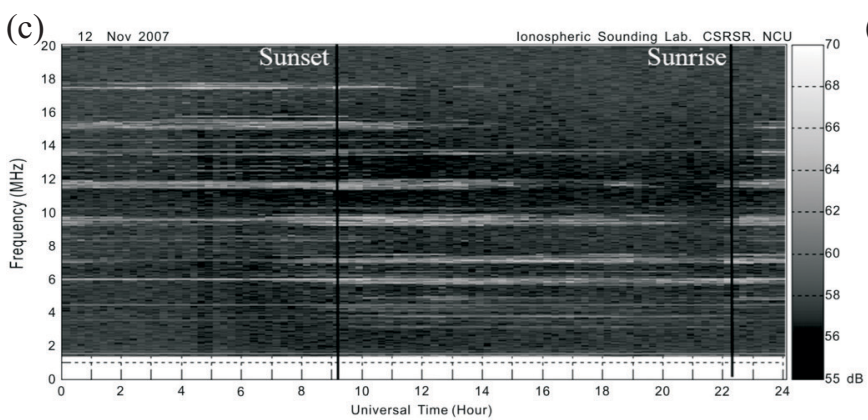

(b)

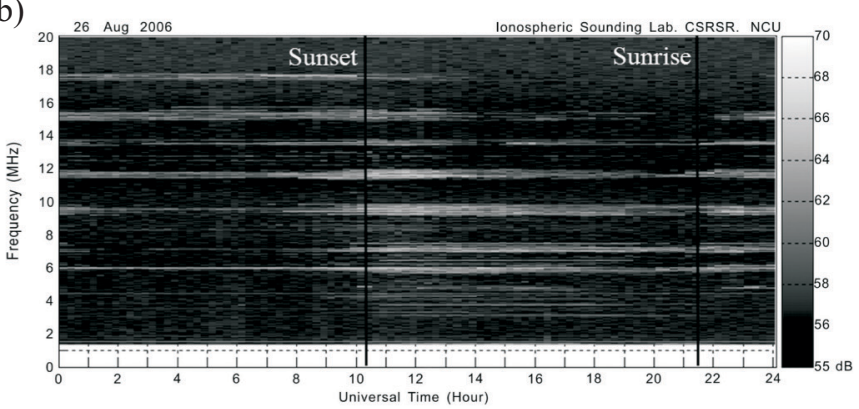

(d)

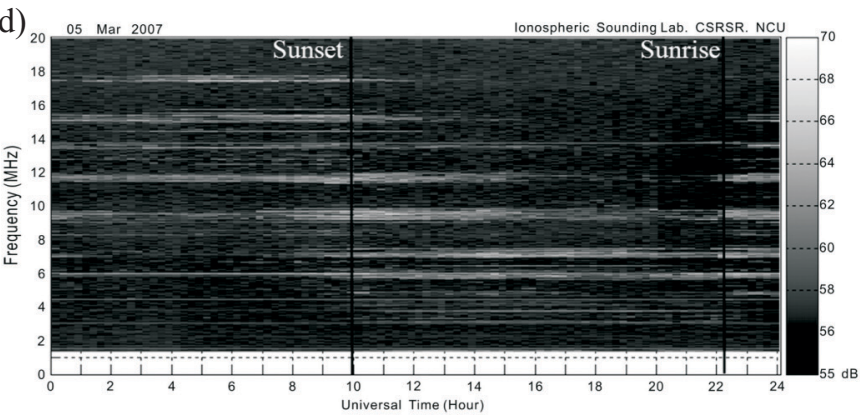

Fig. 13. Typical passive sounding spectrums in four seasons and recorded on: (a) September 12 (fall); (b) August 26 (summer) of 2006; and (c) November 12 (winter); (d) March 5 (spring) of 2007.

\section{CONCLUSIONS}

In this study, we find a cyclical trend that occurs daily from the active and passive sounding results. Information pertaining to RF environment surveillance supplies useful real time statuses for $\mathrm{HF}$ communication and ionospheric observations. For example, we may use the power of background noise to recognize both strong and weak interfer-

\section{REFERENCES}

Berkey, F. T. and M. C. Rose, 1998: AIS PC Engineering Manual. British Antarctic Survey and Utah State University.

Black, J. and T. Y. Hsiao, 2004: FAIS PC32 DSP Upgrade Technical Manual. National Central University, CSRSR, Chung-Li, Taiwan, ROC.

Blagoveshchenskaya, N. F., V. A. Kornienko, A. Brekke, M. T. 
Rietveld, M. Kosch, T. D. Borisova, and M. V. Krylosov, 1999: Phenomena observed by HF long-distance diagnostic tools in the HF modified auroral ionosphere during magnetospheric substorm. Radio Sci., 34, 715-724.

Bowman, G. G. and I. K. Mortimer, 2002: Ionospheric coupling, especially between ionogram-recorded spread-F and sporadic-E enhancements at an equatorial-anomaly crest station, Chung-Li. J. Geophys. Res., 107, 1292.

Bowman, G. G. and I. K. Mortimer, 2003: Spread-F/sporadic E coupling at Chung-Li, especially for postsunset periods of sunspot maximum years. J. Geophys. Res., 108, 1148.

Davies, K., 1990: Ionospheric Radio. Peter Peregrinus Ltd., London.

Howland, P. E., 1994: A passive metric radar using a transmitter of opportunity. In: International Conference on Radar, Soc Elctr. \& Electron, Paris, France, 251-256.

Hsiao, T. Y., 2002: The digital signal processing of Chung-Li Dynasonde. Master Thesis, National Central University, Chung-Li, Taiwan, ROC.

Lind, F. D., J. D. Sahr, and D. M. Gidner, 1999: First passive radar observations of auroral E-region irregularities. Geophys. Res. Lett., 26, 2155-2158.

Mathews, J. D., 1998: Sporadic E: Current views and recent progress. J. Atmos. Solar-Terr. Phys., 60, 413-435.

Pitteway, M. L. V. and J. W. Wright, 1992: Toward an optimum receiving array and pulse-set for the Dynasonde. Radio Sci., 27481-27490.

Ringer, M. A., G. J. Frazer, and S. J. Anderson, 1999: Waveform Analysis of Transmitters of Opportunity for Passive Radar. Tech Rep. DSTO-TR-0809, DSTO Electronics and Surveillance Research Laboratory.

Sahr, J. D. and B. G. Fejer, 1996: Auroral electrojet plasma irregularity theory and experiment: a critical review of present understanding and future directions. J. Geophys. Res., 101, 26893-26909.

Sahr, J. D. and F. D. Lind, 1997: The manastash ridge radar: A passive biostatic radar for upper atmospheric radio science. Radio Sci., 32, 2345.

Tsai, L. C., F .T. Berkey, and G. S. Stiles, 1993: On the derivation of an improved parameter configuration for the Dynasonde. Radio Sci., 28, 785-793.

Vats, H. O., H. Chandra, M. R. Deshpande, and G. D. Vyas, 1995: Radio star and satellite signal scintillation by E region irregularities: A case study. Radio Sci., 30, 475478.

Wright, J. W. and M. L. V. Pitteway, 1979: Real-time data acquisition and interpretation capabilities of the Dynasonde 1, Determination of magnetoionic mode and echolocation using a small spaced receiving array. Radio Sci., 14, $827 \mathrm{pp}$. 